\title{
Formation and Confinement of Wannier Excitons in Free Argon Clusters
}

\author{
J. Wörmer, M. Joppien, G. Zimmerer, and T. Möller \\ II. Institut für Experimentalphysik, Universität Hamburg, D-2000 Hamburg, Federal Republic of Germany
}

(Received 16 April 1991)

\begin{abstract}
The evolution of Wannier excitons in free argon clusters is investigated using fluorescence excitation spectroscopy with synchrotron-radiation excitation. The energetic positions of the absorption bands are analyzed as a function of the cluster size from their appearance in small clusters up to the solid-state limit in crystallites with a radius $R \approx 200 \AA$. A three- and two-dimensional confinement of bulk and surface states is observed.
\end{abstract}

PACS numbers: $71.35 .+\mathrm{z}, 33.20 .-\mathrm{t}, 36.40 .+\mathrm{d}, 78.40 .-\mathrm{q}$

The past years have witnessed great advances in the experimental methods available for the study of atomic and molecular clusters [1]. Experimental investigations are directed in part to the evolution of energy levels from finite to infinite systems. van der Waals clusters, which are bound by dispersion forces, are particularly appealing because their elementary excitations delineate from atomic or molecular states. Direct studies of how these excitations evolve with the size of the system are expected to clarify the connections between atomic or molecular and bulk conceptions of matter [2].

In this Letter we report the first measurements of the evolution of Wannier excitons in free clusters. In particular, the evolution of the energetic position of a solid-state excitation is analyzed from its appearance in small clusters up to the solid-state limit. Wannier excitons are prototypes of electronic excitations in insulating.solids which are characterized by large electron orbits and have no direct atomic parentage [3]. The electron-hole separation exceeds several times the lattice constant of the solid. Therefore the excitations clearly have solid-state character. It is evident that a minimum cluster size is required for the formation of Wannier excitons. Quantum-size effects are predicted for these excitations if the cluster radius becomes comparable to the radius of the electronic orbit [2].

The absorption profiles of Ar clusters containing between 10 and $10^{6}$ atoms are obtained from fluorescence excitation spectra using synchrotron radiation as a light source. In analogy to the solid, the absorption bands are assigned to a hydrogenlike spin-orbit-split exciton series. Tightly bound states with a main quantum number $n=1$ are usually referred to as Frenkel excitons localized at one atom. Additionally, Wannier excitons with a main quantum number up to $n=4$ are observed in rather large clusters. In the solid-state limit, they are characterized by radii $r_{n}=a_{0} n^{2}$ with $a_{0}=1.8 \AA$ [3]. The energetic positions of the absorption bands are close to the wellknown excitonic levels in solid Ar. However, a careful analysis shows that all absorption bands related to Wannier excitons $(n \geq 2)$ are blueshifted compared to the bulk limit. The blueshift is interpreted in terms of a confinement of the electronic excitation in the cluster. In this description the blueshift is a direct consequence of the enhancement of the kinetic energy ("zero-point energy"). The blueshift depends on the number of dimensions in which the confinement takes place. In particular, the confinement energy of surface states is smaller than that of bulk states.

The measurements were performed at the experimental station Clulu behind the high-intensity beam line Superlumi [4] at HASYLAB (Hamburg). In brief, Ar clusters are generated in a nozzle expansion of pure $\mathrm{Ar}$ gas. Rather large clusters are obtained using conical nozzles (diameter between 80 and $500 \mu \mathrm{m}$, opening cone angle $2 \Theta$ between $6^{\circ}$ and $30^{\circ}$ ) at low temperature $(120 \mathrm{~K})$ and a stagnation pressure between 0.3 and 6 bars. After passing a skimmer the cluster beam crosses the beam of monochromatized synchrotron radiation $(75-150 \mathrm{~nm}$, $\Delta \lambda=0.25 \mathrm{~nm}$ ). The size distribution in the cluster beam is analyzed with a time-of-flight mass spectrometer in a different set of measurements [5]. Typically, the width of the size distribution $\Delta N$ corresponds to the average number $N$ of atoms per cluster. The fluorescence light is detected undispersed with either a closed-channel-plate detector (CsTe photocathode, cutoff wavelength $112 \mathrm{~nm}$ ) or a photomultiplier coated with sodiumsalycilate as converter material. Fluorescence excitation spectra are recorded by scanning the monochromator. The spectra recorded with the two different detectors are nearly identical, indicating that the amount of radiation emitted at a wavelength shorter than $112 \mathrm{~nm}$ is small. Therefore, in most cases the channel-plate detector is used because of the low dark counting rate.

Figure 1 shows fluorescence excitation spectra of $\mathrm{Ar}$ clusters containing between 15 and $10^{6}$ atoms. They cover the energy range from the lowest excitation up to the ionization limit. Below the ionization limit the fluorescence yield roughly corresponds to the absorption coefficient because dark relaxation channels are of minor importance [3,6]. Apart from a few weak bands [7] there is a 1:1 correspondence to the absorption bands of solid Ar [8]. In particular, the $n=1$ (Frenkel-type) excitons are clearly visible. With increasing cluster size Wannier excitons $(n \geq 2)$ are formed at the expense of a broad continuum located close to the band-gap energy $E_{g}$ of the solid. This continuum is assigned to Rydberg states in analogy to corresponding absorption bands in the dimer 


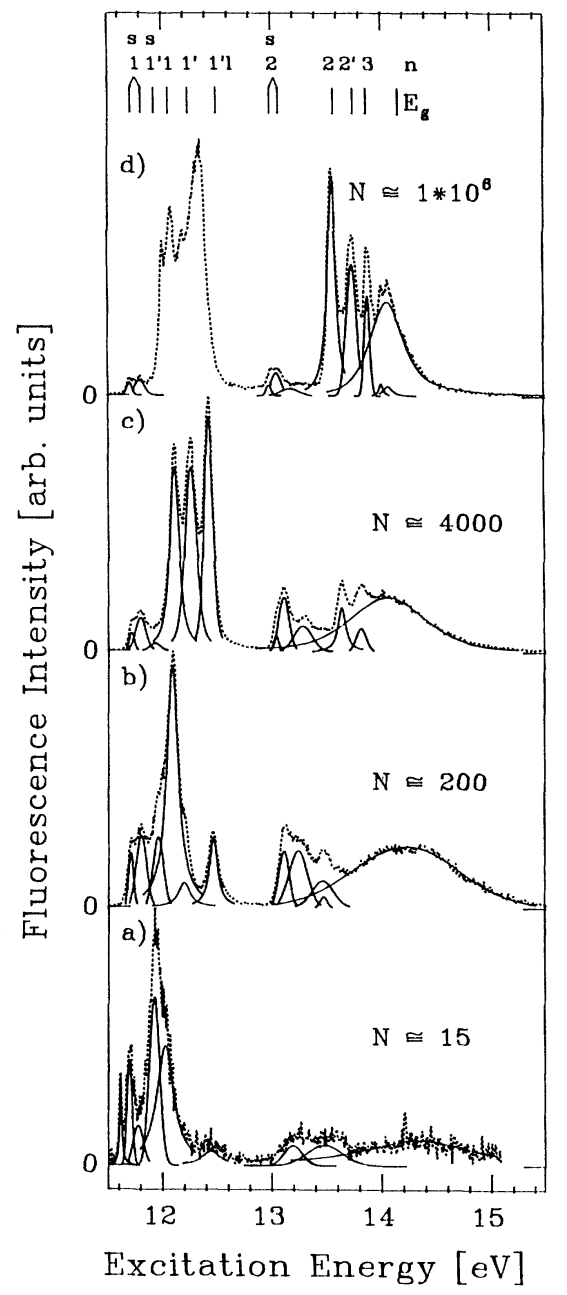

FIG. 1. Fluorescence excitation spectra $(\ldots$.$) and fitted con-$ tributions ( - ) of Ar clusters containing between 15 and $10^{6}$ atoms. The energetic positions of the exciton bands of the solid and the band-gap energy $E_{g}$ are indicated at the top of the figure [22]. Surface states are denoted by $s$, the longitudinal branch of the $n=l^{\prime}$ bulk exciton by $l$.

$[9,10]$.

For the $n=1$ and 2 exciton states, bulk and surface $s$ excitations are clearly separated. The surface states are located on the lower-energy side of the bulk excitons and are identified by a comparison with surface states in solid Ar [8]. The $n=1$ and 1' (the prime denotes the spinorbit partner) excitons are split into transverse and longitudinal branches as already discussed in the case of $\mathrm{Kr}$ clusters [11].

The intensity of the excitonic absorption bands depends sensitively on the cluster size and is directly related to the number of atoms in the interior of the cluster, which are separated from the cluster surface by a dead layer of thickness $d_{n}$. The thickness of this layer is approximately equal to the radius of the corresponding exciton with the

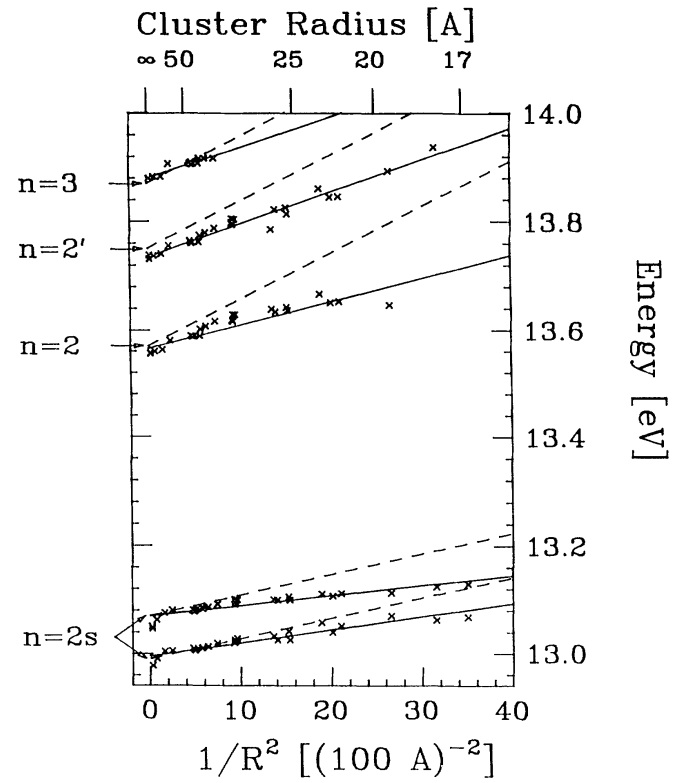

FIG. 2. Energetic positions of the $n=2$ surface $s$ and $n=2$, $2^{\prime}$, and 3 bulk excitons of $\mathrm{Ar}$ clusters as a function of $1 / R^{2}$, where $R$ is the cluster radius. The energetic position of the excitons of solid Ar are indicated in the figure by arrows [22]. The solid lines represent linear fits of the experimental points. Model calculations in the strong confinement limit assuming an effective electron mass of 0.42 free-electron mass and an effective hole mass of 5.3 free-electron masses are given by dashed lines. For the calculation of the surface states, see text.

main quantum number $n[9,12]$. Consequently, the (bulk) Wannier excitons that are related to atoms in the interior of the cluster show up if the radius of the cluster is somewhat larger than the radius of the exciton. More quantitatively, the $n \geq 2$ excitons show up if the cluster radius is 2-3 times larger than the corresponding Wannier radius $r_{n}[9,12]$.

In the following, the discussion focuses on the energetic positions of the excitonic absorption bands. It turned out that the strongest bands related to the $n=1$ excitons show a rather complicated behavior that differs remarkably from that of the Wannier excitons $(n \geq 2)$. In particular, blueshifts and redshifts, respectively, are observed for the longitudinal and transverse branches of the $n=1$ and $n=1^{\prime}$ excitons [12]. However, a precise determination of the maxima of absorption and a detailed analysis of the energetic shifts is somewhat hindered due to saturation effects. In large clusters they become important because of the large oscillator strength of the $n=1$ excitons.

The energies of Wannier excitons $(n \geq 2)$ are characterized by a pronounced blueshift relative to the solidstate limit. Experimentally determined transition energies of the bulk and surface Wannier excitons are displayed in Fig. 2. In view of theoretical considerations they are plotted as a function of $1 / R^{2}$, where $R$ is the 
cluster radius. For the largest clusters the transition energies are within the error bars identical with the corresponding energies of the macroscopic solid, which are indicated by arrows. In other words, the evolution of Wannier excitons is monitored from the appearance in small clusters up to the solid-state limit.

The blueshift is a direct consequence of the confinement of the electronic excitation inside the cluster which leads to an enhancement of the kinetic zero-point energy. Corresponding processes are discussed in great detail for excitons in semiconductor crystallites [13-17]. In a crude approximation $[13,18]$, the energetic shift $\Delta E$ relative to the solid-state value which is interpreted as a confinement energy depends on the relationship between the exciton radius $r_{\mathrm{ex}}$ and the cluster radius $R$. If the cluster radius is much larger than the exciton radius, the exciton is confined as a whole and the energetic shift is given by

$$
\Delta E=h^{2} / 8 R^{2} M \quad \text { (weak confinement), }
$$

where $M=m_{e}+m_{h}$ is the mass of the exciton which is the sum of the effective electron mass $m_{e}$ and the hole mass $m_{h}$. For very small clusters $\left(R \ll r_{\mathrm{ex}}\right)$ the electron and hole are confined separately and the exciton mass $M$ has to be replaced by the reduced exciton mass $\mu=\left(1 / m_{e}+1 / m_{h}\right)^{-1}$; that means the electron and the hole are confined separately:

$$
\Delta E=h^{2} / 8 R^{2} \mu \quad \text { (strong confinement). }
$$

In the case of solid Ar, due to $m_{h} \gg m_{e}, \mu$ can be replaced by $m_{e}$. The two different cases are usually called the weak and strong confinement (or size quantization) limits $[13,15]$, respectively. The influence of the confinement on the correlation between the electron and the hole in small crystallites is considered in several articles $[15,16]$, in particular, for intermediate-size $\left(r_{\mathrm{ex}} \approx R\right)$ particles. However, it should be pointed out that free rare-gas clusters differ remarkably from small crystallites with a large work function embedded in a matrix. Usually, it is assumed that the potential outside the small crystallite is infinite $[14,16]$. In the case of free Ar clusters, however, the electron can easily leave the cluster. It is only impeded by a potential barrier at the surface with a height of a few hundred meV, which arises from polarization effects [19]. Therefore, Eqs. (1) and (2) may serve only as a guideline for the interpretation of the experimental data. In particular, this simple approach is instructive for the comparison between bulk and surface states. In the case of bulk excitons, a more quantitative discussion that takes into account the actual potential and hence changes in the correlation energy is presented below.

The experimental points in Fig. 2 follow straight lines at least for large clusters. Calculated transition energies assuming the case of strong confinement ( $\mu=0.42$ freeelectron mass [3]) are given for the different excitons by dashed lines. The experimentally determined and calcu-
TABLE I. Slope $\left(\mathrm{eV} \AA^{-2}\right)$ of the $1 / R^{2}$ dependence of the energetic positions of the Wannier excitons in Ar clusters in comparison with numerical values for the weak and strong confinement limits according to Eqs. (1) and (2), assuming $m_{e}=0.48$ free-electron mass and $m_{h}=5.3$ free-electron masses.

\begin{tabular}{llcc}
\hline \multicolumn{1}{c}{ Exciton } & Expt. & $\begin{array}{c}\text { Strong } \\
\text { confinement }\end{array}$ & $\begin{array}{c}\text { Weak } \\
\text { confinement }\end{array}$ \\
\hline$n=2$ surface $(12.99 \mathrm{eV})$ & 25 & 38.6 & 4.15 \\
$n=2$ surface $(13.05 \mathrm{eV})$ & 19 & 38.6 & 4.15 \\
$n=2$ bulk & 49.4 & 85.4 & 6.51 \\
$n=2$ ' bulk & 59.5 & 85.4 & 6.51 \\
$n=3$ bulk & 55.9 & 85.4 & 6.51 \\
\hline \hline
\end{tabular}

lated slopes are collected in Table I. It is obvious that the experimental data are much closer to the strong confinement limit than to the weak confinement limit although the cluster radius $R$ is 2-20 times larger than the radius of the $n=2$ exciton $\left(r_{2}=7.2 \AA[3]\right)$. This seems to be in contrast to the theoretical considerations mentioned above. However, the general trend that strong confinement should be observed even in particles relatively large (up to $R / r_{\mathrm{ex}} \approx 10$ ) compared to the exciton is predicted in recently performed calculations for quantum boxes [15]. More precisely, these calculations predict that the energetic shift in particles rather large compared to the exciton is somewhat lower than expected in the strong confinement limit, in qualitative agreement with the experimental results [20]. The reduction of the energetic shift is mainly a consequence of an increase of the Coulomb interaction if the electron and the hole are restricted to move within a small volume [15].

For a more quantitative description, the binding energy of an electron in a positively charged cluster is calculated. For simplification, it is assumed that the hole is fixed at the cluster center, which might be a reasonable assumption [18]. The potential acting on the electron consists of a Coulomb part and polarization terms [14,19] which give rise to a surface barrier of approximately $0.2 \mathrm{eV}$ for a surface layer thickness of $1 \mathrm{ML}$ according to Ref. [19]. Binding energies and transition energies are obtained by solving the radial part of the Schrödinger equation [21]. For electron-hole separations smaller than $R$, the freeelectron mass is replaced by the reduced exciton mass $\mu$. The reduced exciton mass is chosen instead of the effective electron mass to account for the finite effective hole mass. By a choice of reasonable boundary conditions the transition energies of Wannier excitons $(n \geq 2)$ converge to the solid-state values for $R \rightarrow \infty$. Figure 3 displays a comparison between transition energies of the $n=2$ exciton calculated in this way and the experimental results. In particular, for small clusters, a good quantitative agreement with the experimental findings is observed. A comparison with the results in the strong confinement limit according to Eq. (2) shows that the deviations are 


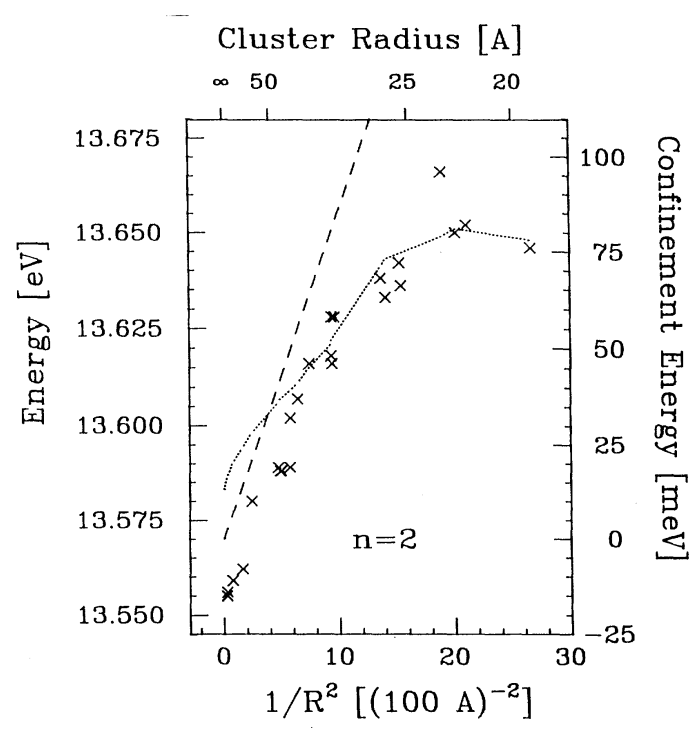

FIG. 3. Energetic position of the $n=2$ exciton in Ar clusters as a function of $1 / R^{2}$, where $R$ is the cluster radius. ( $\left.\times\right)$ Experimental points; (..-) calculated in the strong confinement limit according to Eq. (2); ( ...) model calculation, which takes into account the actual electrostatic potential (see text).

essentially removed if the actual shape of the potential is considered.

As already mentioned, the $n=2$ surface excitons exhibit a somewhat different behavior. In this particular case the electronic excitation is forced to move in the surface [22] or more precisely in the outer shells of the cluster [10]. For a crude estimate of the energetic shift according to Eq. (2) the effective electron mass $m_{e}$ may therefore be replaced by the arithmetic average between the bulk effective electron mass and the free-electron mass. Furthermore, the confinement takes place only in two dimensions because the exciton is already restricted to move in the surface. Under the assumption that the confinement energy is proportional to the number of dimensions in which it takes place, the confinement energy for surface states should be approximately $\frac{2}{3}$ of the one of bulk excitons. The slopes in the $1 / R^{2}$ dependence of the surfaces states given in Table $I$ are calculated under this assumption. Again, the experimentally derived slopes are somewhat smaller than the estimated ones. However, the general trend that the energetic shift of $n=2$ surface excitons is approximately 2 times smaller than the shift of the corresponding bulk excitons is well reproduced in the experimental data (see Fig. 2 and Table I). It is therefore expected that a good quantitative agreement with the experimental data would be achieved if the actual shape of the potential were considered. However, in the case of surface states the calculation is much more complicated because the radial symmetry is lost.

In conclusion, the evolution of Wannier excitons in free clusters is investigated from the appearance of the excitons in small clusters up to the solid-state limit in medium-size crystallites with a radius of approximately 200 $\AA$. The energetic shift of the excitons compared to the macroscopic solid is interpreted in terms of a separate confinement of the electron and the hole inside the cluster. Bulk and surface states exhibit a somewhat different behavior. This is a consequence of the change from three- to two-dimensional confinement of the excitation.

Financial support from the Bundesministerium für Forschung und Technologie (BMFT) under Grant No. 05405 AX B TP 2 is kindly acknowledged.

[1] Proceedings of the Fourth International Symposium on Small Particles and Inorganic Clusters [Z. Phys. D 12 (1990)].

[2] J. Jortner, D. Scharf, and U. Landman, in Excited-State Spectroscopy in Solids, edited by U. M. Grassono and N. Terzi (North-Holland, Amsterdam, 1987), p. 438.

[3] N. Schwentner, E. E. Koch, and J. Jortner, Electronic Excitations in Condensed Rare Gases, Springer Tracts in Modern Physics Vol. 107 (Springer, Berlin, 1985).

[4] H. Wilcke, W. Böhmer, R. Haensel, and N. Schwentner, Nucl. Instrum. Methods 208, 59 (1983).

[5] R. Müller, Diploma thesis, University of Hamburg, 1990 (unpublished).

[6] J. Wörmer, V. Guzielski, J. Stapelfeldt, and T. Möller, Chem. Phys. Lett. 159, 321 (1989).

[7] M. Joppien, J. Wörmer, and T. Möller (to be published).

[8] V. Saile, M. Skibowski, W. Steinmann, P. Gürtler, E. E. Koch, and A. Kozevnikov, Phys. Rev. Lett. 37, 305 (1976).

[9] J. Wörmer and T. Möller, Z. Phys. D 20, 39 (1991).

[10] J. Wörmer, V. Guzielski, J. Stapelfeldt, G. Zimmerer, and T. Möller, Phys. Scr. 41, 490 (1990).

[11] J. Stapelfeldt, J. Wörmer, and T. Möller, Phys. Rev. Lett. 62, 98 (1989).

[12] J. Wörmer, Ph.D. thesis, Hamburg, Internal Report No. DESY F41 HASYLAB 90-05 1990 (unpublished).

[13] Ai. L. Efros and A. L. Efros, Fiz. Tekh. Poluprovodn. 16, 1209 (1982) [Sov. Phys. Semicond. 16, 772 (1982)].

[14] L. E. Brus, J. Chem. Phys. 80, 4403 (1984).

[15] G. W. Bryant, Phys. Rev. B 37, 8763 (1988).

[16] L. Katsikas, A. Eychmüller, M. Giersig, and H. Weller, Chem. Phys. Lett. 172, 201 (1990).

[17] M. G. Bawendi, W. L. Wilson, L. Rothberg, D. J. Caroll, T. M. Jedjn, M. L. Steigerwald, and L. E. Brus, Phys. Rev. Lett. 65, 1623 (1990).

[18] E. Hanamura, Phys. Rev. B 37, 1273 (1988).

[19] P. Stampfli and K. H. Bennemann, Phys. Rev. A 38, 4431 (1988).

[20] This can be seen in Fig. 3 of Ref. [15].

[21] J. Wörmer, M. Joppien, and T. Möller (to be published).

[22] V. Saile, Appl. Opt. 19, 4115 (1980). 